\title{
MAGNETIC DESIGN OF TRIM EXCITATIONS FOR THE ADVANCED LIGHT SOURCE STORAGE RING SEXTUPOLE*
}

\author{
Steve Marks \\ Advanced Light Source \\ Accelerator and Fusion Research Division \\ Lawrence Berkeley Laboratory \\ University of California \\ Berkeley, CA 94720
}

June 1995

\section{MASTER}

Paper presented at the 14th International Conference on Magnetic Technology, Tampere University of Technology, Finland, June 11-16, 1995

*This work was supported by the Director, Office of Energy Research, Office of Basic Energy Sciences, Materials

Sciences Division, of the U. S. Department of Energy, under Contract No. DE-AC03-76SF00098. 


\section{DISCLAIMER}

This report was prepared as an account of work sponsored by an agency of the United States Government. Neither the United States Government nor any agency thereof, nor any of their employees, make any warranty, express or implied, or assumes any legal liability or responsibility for the accuracy, completeness, or usefulness of any information, apparatus, product, or process disclosed, or represents that its use would not infringe privately owned rights. Reference herein to any specific commercial product, process, or service by trade name, trademark, manufacturer, or otherwise does not necessarily constitute or imply its endorsement, recommendation, or favoring by the United States Government or any agency thereof. The views and opinions of authors expressed herein do not necessarily state or reflect those of the United States Government or any agency thereof. 


\section{DISCLAIMER}

This report was prepared as an account of work sponsored by an agency of the United States Government. Neither the United States Government nor any agency thereof, nor any of their employees, make any warranty, express or implied, or assumes any legal liability or responsibility for the accuracy, completeness, or usefulness of any information, apparatus, product, or process disclosed, or represents that its use would not infringe privately owned rights. Reference herein to any specific commercial product, process, or service by trade name, trademark, manufacturer, or otherwise does not necessarily constitute or imply its endorsement, recommendation, or favoring by the United States Government or any agency thereof. The views and opinions of authors expressed herein do not necessarily state or reflect those of the United States Government or any agency thereof. 


\section{DISCLAIMER}

Portions of this document may be illegible in electronic image products. Images are produced from the best available original document. 


\title{
Magnetic Design of Trim Excitations for the Advanced Light Source Storage Ring Sextupole
}

\author{
S. Marks \\ Lawrence Berkeley Laboratory, 1 Cyclotron Road, Berkeley, California
}

\begin{abstract}
The Advanced Light Source (ALS) storage ring sextupole is a unique multi-purpose magnet. It is designed to operate as a sextupole with three auxiliary trim modes: horizontal steering, vertical steering, and skew quadrupole. A perturbation theory for iron-dominated magnets developed by Klaus Halbach provides the basis for this design. The three trim excitations are produced by violating sextupole symmetry and are thus perturbations of the normal sextupole excitation. The magnet was designed such that all four modes are decoupled and can be excited independently. This paper discusses the use of Halbach's perturbation theory to design the trim functions and to evaluate the primary asymmetry in the sextupole mode, namely, a gap in the return yoke to accommodate the vacuum chamber.
\end{abstract}

\section{INTRODUCTION}

Fig. 1 shows a cross-section view of the ALS sextupole. The magnet is constructed from laminations in three separate sections labeled as A, $\mathrm{B}$, and $\mathrm{C}$ in the figure. The return yoke has an asymmetric gap at the midplane; i.e., it is a $\mathrm{C}$ magnet. This is to allow for vacuum chamber clearance. The same lamination is used for all three sections; the laminations for section $\mathrm{A}$ are sheared to produce the required vacuum chamber gap. Each pole has three coils. The inner coils produce the sextupole excitation. These consist of 15 turns of hollow, water-cooled conductor. The outer coils consist of a single water cooling circuit with two solid core coils of 56 and 28 turns except for poles 2 and 5, for which the larger coil has 54 turns. This modification is to compensate for the effect of the C-magnet asym-

Manuscript received June 13, 1995.

This work was supported by the Director, Office of Energy Research, Office of Basic Energy Sciences, Mat. Sci. Div., of the U.S. Dept. of Energy, Contract No. DEAC03-76SF00098.

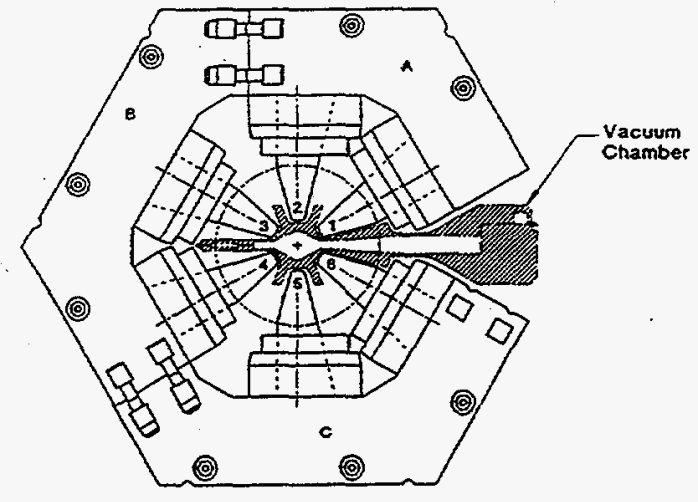

Fig. 1. ALS Storage Ring Sextupole

metry on horizontal steering. This point will be discussed in Section IV.

The ALS sextupole features four primary asymmetries which will be discussed in the sections below: C-magnet asymmetry, and excitation asymmetries associated with horizontal steering, vertical steering, and skew quadrupole. The analysis of these asymmetries is based upon a theory of perturbations in iron core magnets developed by Klaus Halbach [1]. Engineering design calculations are documented in several LBL Engineering Notes [2] [3]. The major parameters of of the magnet are summarized in Table $I$.

TABLE I

ALS Sextupole Parameters

\begin{tabular}{ll}
\hline Parameter & Value \\
\hline Magnet Aperture Radius & $3.5 \mathrm{~cm}$ \\
Good Field Radius & $3.0 \mathrm{~cm}$ \\
Sextupole Field & $500 \mathrm{~T} / \mathrm{m}^{2}$ \\
Sextupole Pole Tip Field & $0.6125 \mathrm{~T}$ \\
Sextupole Amp-Turns & 5868 \\
Horizontal Steering Field & $0.07 \mathrm{~T}$ \\
Horizontal Steering Current & $42.9 \mathrm{Amps}$ \\
Vertical Steering Field & 0.07 \\
Vertical Steering Current & $35.7 \mathrm{Amps}$ \\
Skew Quadrupole Field & $0.75 \mathrm{~T} / \mathrm{m}$ \\
Skew Quadrupole Current & $29.1 \mathrm{Amps}$ \\
\hline
\end{tabular}




\section{Use of Perturbation Theory}

The Halbach theory develops perturbation coefficients for a reference pole centered on the horizontal axis at $\theta=0$. The effect due to errors on a set of poles is calculated from this sensitivity coefficient, the strength of the perturbation, and a geometry factor which accounts for the actual position of the poles. The perturbation to the $n$th harmonic is formulated as

$$
\frac{\left|H_{n}\right|}{\left|H_{N}\right|}=\frac{n}{N} \Delta C_{n}
$$

where $n$ designates the particular harmonic being considered, $N$ designates the fundamental, and $\Delta C_{n}$ is the perturbation to the $n$th multipole coefficient.

$$
\Delta C_{n}=\Delta C_{n}(0) \epsilon \sum_{j} e^{-i n \theta_{j}}
$$

where $\Delta C_{n}(0)$ is the perturbation corresponding to the reference pole, $\epsilon$ is the strength of the perturbation, and the $\theta_{j}$ are the angle locations of pole centers. The summation term in the equation is the geometry factor, $g_{n}$.

Normalized sensitivity coefficients can be calculated directly from a set of equations given in Halbach's paper or from tables which include values for the most important cases. The tables give values for $\frac{n}{N} \frac{\Delta C_{n}(0)}{i \epsilon}$

\section{Effect of C-Magnet Geometry}

A break is made in the return yoke to accommodate the ALS vacuum chamber. This break violates sextupole symmetry and therefore represents a perturbation in the multipole signature of the magnet. In particular; a new set of "unallowed" multipoles will be present that would normally not be present if sextupole symmetry were preserved. This results in a modification in the pole surface scalar potential values, which is equivalent to a perturbation in excitation. The magnitude of the excitation error due to a gap of $2 a$ can be determined. First, assume that the yoke is continuous as indicated by the dashed line in Fig. 2. Now apply Ampere's law to the integral path represented in the figure.

$$
\int H \cdot d l=\frac{1}{3} H_{p} r_{p}+H_{1} l_{1}+H_{2}\left(l_{2}+a\right)
$$

where $H_{p}$ is the pole tip field, $r_{p}$ is the pole tip radius, $H_{1}$ and $H_{2}$ are the average field values

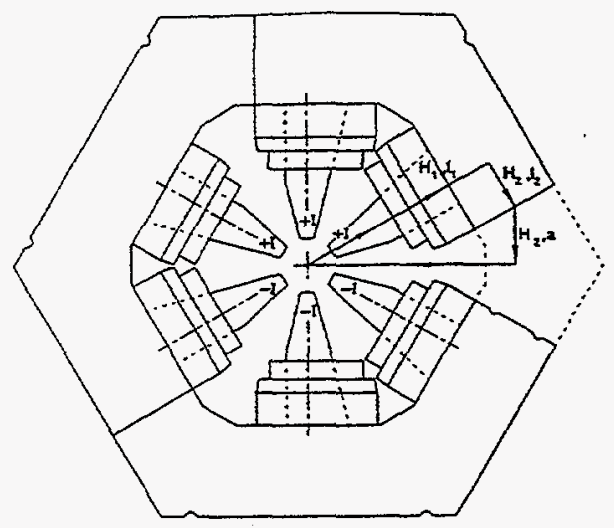

Fig. 2. Perturbation due to C-magnet geometry

in the pole and return yoke, respectively and $l_{1}$ and $l_{2}$ are the respective path lengths. The iron section corresponding to the gap can be removed and replaced by a coil with excitation $\mathrm{H}_{2} a$ without changing the magnitude of the integral in Eq. 3. Therefore it is clear that the magnitude of the excitation error, $I$, resulting from the removal of the iron is

$$
I=H_{2} a
$$

This will result in excitation errors $+I$ at poles 1,2 , and 3 , and $-I$ at poles 4,5 , and 6 .

An important qualitative result is evident from Eq. 4 without any further analysis. The magnitude of the perturbation is proportional to the average field level in the return yoke. Therefore the effect can be kept arbitrarily low by increasing the cross-section of the return yoke. It is particularly important to avoid saturation in the return yoke.

Now let us evaluate the change in multipoles due to $\mathrm{C}$-magnet geometry. The geometry factor, to be inserted into Eq. 2 for this case is

$$
g_{n}=2 i\left[\sin \left(\frac{n \pi}{6}\right) \sin \left(\frac{n \pi}{2}\right) \sin \left(\frac{5 n \pi}{6}\right)\right]
$$

From two dimensional magnetic analysis with the POISSON code, the value of $\mathrm{H}_{2}$ is $4.6 \mathrm{Oe}$ for full sextupole excitation, and the half gap is about $10 \mathrm{~cm}$. This gives a perturbation strength of

$$
\epsilon=\frac{H_{2} a}{N I}=8.09 \times 10^{-3}
$$

The results of calculations are summarized in Table II. Relative multipole strengths are given at the pole tip radius of $3.5 \mathrm{~cm}$. Note that some multipoles are not allowed by symmetry. Only non-zero values are shown in the table. 
TABLE II

Multipoles due to C-MAGnet Geometry

\begin{tabular}{||c|c|c|c||}
\hline $\mathrm{n}$ & $\frac{n}{N} \frac{\Delta C_{n}(0)}{i c}$ & $g_{n}$ & $\frac{\left|H_{n}\right|}{\left|H_{N}\right|} @ 3.5 \mathrm{~cm}$ \\
\hline 1 & $9.79 \times 10^{-2}$ & 4.0 & $2.51 \times 10^{-3}$ \\
3 & $1.67 \times 10^{-1}$ & 2.0 & $2.14 \times 10^{-3}$ \\
5 & $7.09 \times 10^{-2}$ & 4.0 & $1.82 \times 10^{-3}$ \\
7 & $-1.34 \times 10^{-2}$ & -4.0 & $3.43 \times 10^{-4}$ \\
\hline
\end{tabular}

\section{Horizontal SteERING}

Consider the excitations required to produce a vertical dipole used for horizontal steering. Since we want all magnetic modes to be independent, care must be taken so as not to also produce a sextupole field, which would then modify the primary sextupole excitation.

It is clear from symmetry that to produce a vertical dipole, the magnitude of excitations for poles $1,3,4$, and 6 are equal, with that for 4 and 6 being of opposite polarity of that for 1 and 3 . Let us call this $I_{k 1}$. The magnitude of excitation in poles 2 and 5 must also be equal and opposite. Let us call this $I_{h 2}$. We want to set the ratio of the two excitations, $I_{h 1}$ and $I_{h 2}$, to eliminate a sextupole component. The two sextupole contributions are proportional to the two geometry factors. The geometry factors for sextupole contributions, $g_{31}$ and $g_{32}$, corresponding to excitations $I_{h 1}$ and $I_{h 2}$, respectively, are given in Eqs. 7 and 8 below.

$$
\begin{gathered}
g_{31}=e^{i \pi / 2}+e^{i 5 \pi / 2}-e^{-i \pi / 2}-e^{-5 i \pi / 2}=4 i \\
g_{32}=e^{3 \pi / 2}-e^{-3 i \pi / 2}=-2 i
\end{gathered}
$$

Therefore, in order that the horizontal steering mode not produce a sextupole, the strength of $I_{h 2}$ should be double that of $I_{h 1}$. This is easily accomplished by connecting all coils in series and using twice as many turns in the coils for poles 2 and 5 .

Now let us calculate the required value of the excitations. The value of the normalized dipole sensitivity coefficient is 0.0979 . The geometry factor for $n=1$ is

$$
g_{1}=2 i\left(\sin \frac{\pi}{6}+\sin \frac{5 \pi}{6}+2 \sin \frac{\pi}{2}\right)=6 i
$$

Using Eqs. 1 and 2 we can calculate the perturbation, $\epsilon=0.1946$, which multiplied by the normal sextupole excitation gives the required horizontal steering excitation. Therefore the two excitations are: $I_{h 1}=1107$ amp-turns, and $I_{h 2}=2214$ amp-turns. Using this value for $\epsilon$, the full set of multipoles are calculated; values are summarized in Table III.

The condition for sextupole decoupling applies to a magnet which obeys standard sextupole geometry. The asymmetry associated with the $\mathrm{C}$-magnet geometry modifes the requirement. During prototype measurements a two turn reduction in the coil at poles 2 and 5 was empirically determined to effectively remove the sextupole coupling. This effect is analyzed below.

Consider an integration path through the top half of the sextupole. It passes through the return yoke gap, the return yoke, and then passes back along the horizontal midplane. The enclosed current in this loop is zero. Since all field components in the return yoke parallel to this path are in the same direction and there is no contribution from the part of the path along the midplane (symmetry requires that field lines are perpendicular to the midplane), application of Ampere's law produces the following requirement on field in the yoke gap:

$$
H_{g} a=H_{1} l+H_{2} l+H_{3} \frac{l}{2}
$$

where $H_{g}$ is the gap field, $a$ is the half gap, $H_{1}, H_{2}$ and $H_{3}$ are horizontal steering excitation field levels in the return yoke between poles 1 and 2 , between poles 2 and 3 , and pole 3 and the midplane, respectively, and $l$ is the average path length in the return yoke between two poles. $H_{g} a$ represents an excitation perturbation that is equal for each pole.

Since we need to keep the horizontal steering excitation at poles 2 and 5 twice that at the other poles to eliminate sextupole coupling, the value of $H_{g} a$ is the magnitude of the required correction. From Poisson runs typical values for $H_{1}$, $H_{2}$ and $H_{3}$ are $0.7 \mathrm{Oe}, 1.9 \mathrm{Oe}$, and $2.6 \mathrm{Oe}$, respectively for full horizontal steering excitation. The value of $l$ is approximately $30 \mathrm{~cm}$. These result in a value for $H_{g} a$ of $117 \mathrm{Oe}-\mathrm{cm}$, which corresponds to 93 amp-turns. The full current value for the horizontal steering is 43 amps. So, the calculation is in close agreement with the required two turn reduction discovered in the prototype measurements. 
TABLE III

MULTIPOLES FOR HORIZONTAL STEERING MODE

\begin{tabular}{||c|c|c|c||}
\hline $\mathrm{n}$ & $\frac{n}{N} I_{n}$ & $g_{n}$ & $\frac{\left|H_{n}\right|}{\left|H_{N}\right|} @ 3.5 \mathrm{~cm}$ \\
\hline 5 & $7.09 \times 10^{-2}$ & 3.0 & $8.28 \times 10^{-2}$ \\
7 & $-1.34 \times 10^{-2}$ & -3.0 & $1.56 \times 10^{-2}$ \\
11 & $9.72 \times 10^{-3}$ & -3.0 & $-1.14 \times 10^{-2}$ \\
13 & $-1.01 \times 10^{-3}$ & 3.0 & $-1.18 \times 10^{-3}$ \\
\hline
\end{tabular}

\section{VERTICAL STEERING}

Now consider excitation for production of a horizontal dipole field for vertical steering. From symmetry we know that this can be accomplished with an excitation of poles $1,3,4$, and 6 . The magnitude will be equal for all four poles, with the polarity at poles 3 and 4 opposite that at poles 1 and 6 . Poles 2 and 5 are not excited.

We use the same dipole sensitivity coefficient of 0.0979 as for the horizontal steering mode. The geometry factor is

$$
g_{n}=e^{i n \pi / 6}+e^{-i n \pi / 6}-e^{5 i n \pi / 6}-e^{-5 i n \pi / 6}
$$

For $n=1$, the value is 3.464 . Using the same procedure as before, a value of $\epsilon=0.337$ is calclulated. From this, the require excitaion of 1917 amp-turns is determined. The full set of multipole values are summarized in Table IV.

TABLE IV

Multipoles for Vertical SteERING MOde

\begin{tabular}{|c|c|c|c||}
\hline $\mathrm{n}$ & $\frac{n}{N} \cdot L_{n}$ & $g_{n}$ & $\frac{\left|E_{n}\right|}{\left|H_{N}\right|} 3.5 \mathrm{~cm}$ \\
\hline 5 & $7.09 \times 10^{-2}$ & 3.0 & $8.28 \times 10^{-2}$ \\
7 & $-1.34 \times 10^{-2}$ & -3.0 & $1.56 \times 10^{-2}$ \\
11 & $9.72 \times 10^{-3}$ & -3.0 & $1.14 \times 10^{-2}$ \\
13 & $-1.01 \times 10^{-3}$ & 3.0 & $-1.18 \times 10^{-3}$ \\
\hline
\end{tabular}

for this excitaion is

$$
g_{n}=e^{i n \pi / 2}+e^{-i n \pi / 2}=2 \cos \frac{n \pi}{2}
$$

For $n=2$ the value is -2 . For the required field gradient of $0.75 \mathrm{~T} / \mathrm{m}$, the pole tip field is $0.02625 \mathrm{~T}$. Using Eqs. 1 and 2 we determine a perturbation coeficient value of 0.1374 . The required excitation is then 781 amp-turns. The full set of multipoles are summarized in Table V.

TABLE V

MUlTipoles For SKEW QUADRUPOLE MODE

\begin{tabular}{||c|c|c|c||}
\hline $\mathbf{n}$ & $\frac{n}{N} J_{n}$ & $g_{n}$ & $\frac{\left|H_{n}\right|}{\left|H_{N}\right|} @ 3.5 \mathrm{~cm}$ \\
\hline 4 & $1.33 \times 10^{-1}$ & 2.0 & $3.65 \times 10^{-2}$ \\
8 & $-1.07 \times 10^{-2}$ & 2.0 & $-2.94 \times 10^{-3}$ \\
10 & $9.13 \times 10^{-3}$ & -2.0 & $-2.51 \times 10^{-3}$ \\
\hline
\end{tabular}

\section{Conclusion}

Trim modes can be produced in a magnet by excitations that violate the magnet's natural symmetry. Halbach's theory of perturbations of iron core magnets [1] provides an indispensable design tool for accomplishing this. Magnetic measurements of the ALS sextupole demonstrated that the trim functions operated as expected from the analysis. A total of $48 \mathrm{mag}-$ nets of this type have been installed and are currently operating successfully in the ALS storage ring.

\section{REFERENCES}

[1] K. Halbach, "First Order Perturbation Effects in Iron-Dominated Two-Dimensional Symmetrical Multipoles", Nuclear Instruments \& Methods, Vol. 74, 1969.

[2] S. Marks, "Multipoles for ALS Ring Sextupole", LBL Engineering Note M6599A, 1987.

[3] S. Marks, "Design Calculations for Storage Ring Sextupole", LBL Engineering Note M6671, 1987.

\section{Skew Quadrupole}

A skew quadrupole mode is produced by excitation of poles 2 and 5 with the same magnitude and polarity. The normalized quadrupole sensitivity coefficient is 0.156 . The geometry factor 